\title{
Estrutura populacional e incremento corrente anual de casca-de-anta (Drimys brasiliensis Miers - Winteraceae) em Caçador, Santa Catarina, Brasil
}

\author{
MARIOT, A. ${ }^{\text {; }}$ MANTOVANI, A. ${ }^{2}$; BITTENCOURT, R. ${ }^{1}$; FERREIRA, D.K. ${ }^{1}$; REIS, M.S. ${ }^{1 *}$ \\ ${ }^{1}$ Núcleo de Pesquisas em Florestas Tropicais, Universidade Federal de Santa Catarina, Caixa Postal 476, CEP: \\ 88040-900, Florianópolis-Brasil ${ }^{2}$ Universidade do Estado de Santa Catarina, Av. Luiz de Camões, 2090, Bairro \\ Conta Dinheiro, CEP:88520-000, Lages-Brasil*msreis@cca.ufsc.br
}

\begin{abstract}
RESUMO: Drimys brasiliensis Miers, conhecida como cataia ou casca-de-anta, é árvore nativa da Mata Atlântica e as cascas tem sido utilizadas medicinalmente a partir da exploração de plantas em populações naturais. Este trabalho teve como objetivo estudar a demografia de duas populações naturais de $D$. brasiliensis, (1) Reserva Genética Florestal de Caçador (RGFC) e (2) Floresta Nacional de Caçador (FLONA), ambas no município de Caçador (SC), visando fundamentar estratégias de exploração sustentável e conservação deste recurso florestal não-madeireiro. $A$ maior luminosidade no sub-bosque existente na FLONA atuou positivamente na espécie, aumentado a capacidade de incremento em diâmetro à altura do peito (DAP), altura $(H)$ e área basal $(A B)$, além de antecipar a reprodução em plantas de menor porte. A análise do padrão espacial das plantas reprodutivas na RGFC mostrou padrão agregado, e na FLONA até a distância de 25 metros. Esse comportamento indicou que a espécie apresenta potencial de manejo em florestas secundárias, formação sucessional em que se encontra a maioria dos remanescentes de Mata Atlântica, além de indicar potencial para cultivo em sistemas agroflorestais.
\end{abstract}

Palavras-chave: Drimys brasiliensis, demografia, exploração de cascas

\begin{abstract}
Population structure and annual increment in "casca-de-anta" (Drimys brasiliensis Miers - Winteraceae) from Caçador Municipality, Santa Catarina State, Brazil. Known as "cataia" or "casca-de-anta", Drimys brasiliensis Miers is a tree native to the Atlantic Forest and its barks have been medicinally used by exploring natural populations. The present work aimed to study the demography of two $D$. brasiliensis natural populations, (1) Caçador Forest Genetic Reserve (RGFC) and (2) Caçador National Forest (FLONA), both located in Caçador Municipality, Santa Catarina State, Brazil, in order to establish strategies for sustainable exploration and conservation of such non-timber forest resource. The higher luminosity in FLONA understory was beneficial to the species by increasing their increment capability regarding diameter at breast height $(\mathrm{DBH})$, height $(\mathrm{H})$ and basal area $(\mathrm{BA})$, and made the reproduction in smaller plants earlier. The spatial analysis of reproductive plants in RGFC had an aggregate pattern, whereas in FLONA until $25 \mathrm{~m}$ distance was detected. Such results indicated that this species presents potential for management in secondary forests, the successional formation where the major Atlantic Forest remnants are found, as well as potential for cultivation in agroforestry systems.
\end{abstract}

Key words: Drimys brasiliensis, demography, bark exploration

\section{INTRODUÇÃO}

O conhecimento da demografia das espécies vegetais em populações naturais é imprescindível para o estabelecimento de estratégias de conservação e exploração que sejam sustentáveis (Fantini et al., 1992; Hall \& Bawa, 1993; Reis, 1996; Puchalski et al., 2006; Ruschel et al., 2006), além de possibilitar testar se as metodologias em uso são ou não sustentáveis (Degen et al., 2006; Wadt et al., 2008; Sebben et al., 2008).

A partir dos estudos demográficos, juntamente com outros estudos de autoecologia, diversos autores propõem estratégias de manejo buscando a sustentabilidade em florestas tropicais.

Recebido para publicação em 16/01/09

Aceito para publicação em 31/01/10

Rev. Bras. PI. Med., Botucatu, v.12, n.2, p.168-178, 2010. 
Reis et al. (2000) propuseram para o manejo do palmiteiro (Euterpe edulis) na Mata Atlântica a permanência de 60 plantas reprodutivas por hectare para a manutenção da dinâmica demográfica e da estrutura genética. Hernández-Apolinar et al. (2006) verificaram que a exploração de oito árvores por hectare por ano é sustentável para Bursera glabrifolia no México. Wadt et al. (2008) verificaram que a coleta de castanha (Bertholletia excelsa) em Reservas Extrativistas (RESEX) na Amazônia possibilitava a regeneração natural da espécie.

No caso das florestas secundárias, estágio sucessional em que se encontra a maioria dos remanescentes de Mata Atlântica, a sua estrutura possibilita a entrada de maior quantidade de luz no interior da floresta em relação às florestas primárias, propiciando condições semelhantes às encontradas nas clareiras ocorrentes em florestas primárias. Com isso, a dinâmica demográfica das espécies pode sofrer modificações, como a regeneração natural e o incremento de algumas espécies de clareira. É o caso de Piper cernuum, espécie medicinal típica de clareiras, que em condições de floresta secundária produz mais de 80 vezes a quantidade de biomassa do que em floresta primária, resultando em maior potencial produtivo (Mariot et al., 2007). No caso de Drimys brasiliensis, a espécie chega a ser dominante em algumas florestas primárias, porém dependente de clareiras para a regeneração (Smith-Ramírez \& Amesto, 1994).

Drimys brasiliensis Miers, conhecida como cataia ou casca-de-anta, é árvore nativa da Mata Atlântica e as cascas têm sido utilizadas medicinalmente a partir da exploração de plantas em populações naturais. Porém, não existem estratégias de manejo de populações naturais sustentáveis. Ocorrem no Brasil, nos Estados da Bahia, Minas Gerais, Espírito Santo, Rio de Janeiro, São Paulo, Paraná, Santa Catarina e Rio Grande do Sul (Trinta \& Santos, 1997).

$D$. brasiliensis apresenta diversas utilidades, fornecendo madeira amarelada com largas veias róseas, às vezes castanho-clara, sempre firmes e fáceis de trabalhar, prendendo bem os pregos, porém pouco resistente (obras internas), carpintaria e caixotaria, lenha e carvão. Indicada para paisagismo de pequenos espaços (Trinta \& Santos, 1997; Backes \& Irgang, 2002). A casca é aromática utilizada como estomáquica, antiescorbútica, anti-diarréica, sudorífica e tônica. A casca das espécies de Drimys era comercializada como "Casca de Winter" ou "Córtex Winteranus" da Farmacopéia Universal ("Ecorce de Winter" dos franceses, "Winter's bark" dos Angloamericanos), descoberta casualmente pelo capitão Winter, um dos tenentes do navegador Sir Francis
Drake, quando, em 1577, uma tempestade o obrigou a refugiar-se no Estreito de Magalhães, para tratar a tripulação de escorbuto (Trinta \& Santos, 1997).

Pio Corrêa (1931) relata que os aborígenes Araucanos, celebravam sob a copa de uma das espécies do gênero, ritos e festas, estando associada a mitologia deste povo. O povo comenta que a anta (Tapirus americanus), quando doente, recorre a cascas desta árvore, assim justificando o nome vernáculo da espécie como casca-d'anta. Em Santa Catarina é utilizada, na região do Planalto Catarinense, como condimento para carnes, transformando suas cascas em pó e utilizando-a como substituto da pimenta-do-reino (Trinta \& Santos, 1997).

Popularmente, Drimys brasiliensis é internamente utilizada como estimulante, antiespasmódica, aromática, antidiarréica, antifebril, contra hemorragia uterina e em certas afecções do trato digestivo (Simões et al., 1986). Segundo estes autores, na literatura sobre a espécie são indicados vários compostos químicos, sendo que das cascas isolou-se sesquiterpenos, terpenóides e lignanas; das folhas, terpenóides e flavonóides com ação antitumoral e nas folhas jovens foram detectadas substâncias cardioativas, e na utilização farmacêutica é comercializada sob as formas de tintura e elixir, indicados em distúrbios do trato digestivo e como antifebril. Malheiros et al. (2005) detectaram atividade antifúngica dos sesquiterpenos encontrados nas cascas de $D$. brasiliensis. Cechinel Filho et al. (1998) isolaram e identificaram diversos compostos ativos a partir das cascas de $D$. brasiliensis, e verificaram que o polygodial era mais potente no controle da dor do que a aspirina. Ribeiro et al. (2008) verificaram que os óleos essenciais encontrados nas folhas e cascas de $D$. brasiliensis eram letais para carrapatos de gados e cachorros.

O objetivo deste trabalho foi a realização de estudos demográficos em duas populações naturais de Drimys brasiliensis no município de Caçador (SC), visando fundamentar estratégias de exploração sustentável e conservação deste recurso florestal não-madeireiro, seguindo a concepção de sistema de manejo em regime de rendimento sustentado proposta em Fantini et al. (1992) e aplicado para o palmiteiro - Euterpe edulis (Reis et al., 2000; Conte et al., 2008), para a pariparoba - Piper cernuum (Mariot et al., 2002; 2003; 2007), para a espinheira-santa Maytenus ilicifolia (Steenbock et al., 2003; Steenbock \& Reis, 2004), para o caraguatá Bromelia anthiacantha (Duarte et al., 2007) e para a samambaia-preta - Rumohra adiantiformis (Baldauf et al., 2007). 


\section{MATERIAL E MÉTODO}

Para os estudos da demografia das populações naturais de casca-de-anta (Drimys brasiliensis Miers - Winteraceae) foram instaladas três parcelas permanentes de 1 hectare cada (100 x 100 metros), sendo duas na Reserva Genética Florestal de Caçador (RGFC) e uma na Floresta Nacional de Caçador (FLONA). Todas as parcelas foram subdivididas em subparcelas de $100 \mathrm{~m}^{2}$ (10 x 10 metros), sendo que em cada vértice inferior esquerdo das mesmas foi colocada uma estaca de alumínio contendo o número da subparcela.

A RGFC está localizada no município de Caçador a aproximadamente $6 \mathrm{~km}$ do centro da cidade. É uma Reserva Particular do Patrimônio Natural (RPPN), administrada pela Empresa de Pesquisa Agropecuária e Extensão Rural de Santa Catarina - EPAGRI e pela Empresa Brasileira de Pesquisa Agropecuária - EMBRAPA. A Reserva possui uma área total de $772 \mathrm{ha}$. O clima da região é do tipo Cfb de Köppen (mesotérmico, úmido sem estação seca definida, verões frescos, com ocorrência de geadas severas e freqüentes no inverno) e o relevo da região é suavemente ondulado a ondulado. Essa área foi escolhida por ser considerada uma das poucas áreas de grande representatividade da Floresta Ombrófila Mista em bom estado de conservação. As coordenadas do local de estudo são $26^{\circ} 51^{\prime} 0,81^{\prime \prime} S$ e 5057'58,12'O.

A FLONA (ICMBio), está localizada no Distrito de Taquara-Verde, a $26 \mathrm{~km}$ da cidade de Caçador, na Rodovia 451, Km 26. Possui uma área de 710 ha, o clima é do tipo Cfb, ou seja, clima mesotérmico, subtropical úmido, com verões frescos, sem estação seca definida e geadas severas frequentes. A vegetação original caracterizava-se por Floresta Ombrófila Mista, porém hoje a totalidade da FLONA é composta por reflorestamentos de Pinus spp. e Araucaria angustifolia, sendo que esses últimos ocupam uma área de 270 ha. Aárea de estudo na FLONA é um talhão com reflorestamento de araucária implantado em 1958 com sub-bosque com presença de $D$. brasiliensis. As coordenadas do local de estudo são 2645'50,24'S e 5111'59,30"O.

Todas as plantas encontradas dentro das parcelas foram avaliadas em março de 2004 e reavaliadas em março de 2005, 2006, 2007 e 2008. As plantas com altura total superior a $1,30 \mathrm{~m}$ foram avaliadas quanto ao diâmetro à altura do peito (DAP) (centímetros), utilizando-se paquímetro florestal, e quanto à altura total com régua dendrométrica (metros). As plantas com altura total inferior a 1,30 $\mathrm{m}$ foram medidas quanto à altura total com régua dendrométrica (metros) e a planta considerada dentro da classe de regeneração natural.

Todas as plantas foram avaliadas quanto à fenologia reprodutiva e etiquetadas com placas de alumínio contendo o número da sub-parcela e da planta, além do mapeamento através de coordenadas $X$ e $Y$. Foram estimados os incrementos em diâmetro à altura do peito (DAP) e altura, além da mortalidade e da entrada de plantas novas (avaliadas pela primeira vez, resultantes da regeneração natural oriunda da germinação das sementes no ano anterior) nas áreas estudadas em todos os anos avaliados. A parcela 1 da RGFC está inclusa numa parcela de $170 \times 300$ metros (5,1 hectares), na qual também foram mapeadas todas as plantas reprodutivas para análise do padrão espacial. Os dados referentes as duas parcelas da RGFC foram analisados a partir de estatísticas descritivas.

A partir dos dados coletados nas cinco avaliações (2004 a 2008) foram testados modelos matemáticos para expressar o comportamento do incremento corrente anual médio em relação ao $\mathrm{DAP}$, altura total e área basal ( $\mathrm{AB})$, através de análises de regressão. Para a análise do padrão espacial da parcela 1 da RGFC e da FLONA foi utilizado a Função K de Ripley (1977). O raio (h) utilizado para o cálculo do $\mathrm{K}(\mathrm{h})$ foi de $1 \mathrm{~m}$. Os limites de confiança para a função univariada foram criados utilizando 499 simulações de eventos em completa aleatoriedade espacial. Tanto a função $\mathrm{K}$ quanto seus intervalos de confiança foram calculados utilizando-se algoritmos escritos no "R pacote splancs" (Rowlingson \& Diggle, 1993).

\section{RESULTADO E DISCUSSÃO}

Os resultados dos levantamentos demográficos estão expressos em número de indivíduos por hectare na Tabela 1. Na RGFC, para o ano de 2004, o número total médio de plantas por hectare foi de 195, sendo $92 \mathrm{com}$ altura superior a $1,3 \mathrm{~m}$, das quais 35 eram reprodutivas, além de $103 \mathrm{com}$ altura inferior a 1,3 m. De 2004 a 2008 foram recrutadas 24 plantas e morreram 46 , conforme dinâmica expressa na Tabela 1. O número médio de ramos por planta foi de 1,1. A definição dos indivíduos reprodutivos foi através da visualização de alguma das fenofases reprodutivas no momento das avaliações (botões florais, flores e frutos).

Das 46 plantas que morreram, três tinham altura superior a $1,3 \mathrm{~m}(1,5 \mathrm{~m}, 2,0 \mathrm{~m}$ e $16 \mathrm{~m})$, das quais apenas uma era reprodutiva ( $16 \mathrm{~m}$ de altura e 20,1 cm de DAP), que morreu devido a quebra da copa causada por queda de árvore, sendo as 43 restantes pertencentes à classe de regeneração natural ( $<1,3$ metros de altura).

O DAP médio em 2004 foi de $7,94 \mathrm{~cm}$ considerando todas as plantas com altura superior a 1,3 m, passíveis de serem avaliadas, e de 12,46

Rev. Bras. Pl. Med., Botucatu, v.12, n.2, p.168-178, 2010. 
TABELA 1. Número de plantas, mortalidade e recrutamento de Drymis brasiliensis na região de Caçador, SC, expressos por hectare.

\begin{tabular}{lcccc}
\hline Parâmetros & $\begin{array}{c}\text { Reserva Genética } \\
\text { Florestal de Caçador }\end{array}$ & $\begin{array}{c}\text { Floresta Nacional } \\
\text { de Caçador }\end{array}$ \\
\hline Número total de plantas & \multicolumn{2}{c}{195} & 467 \\
Número de plantas com altura $<1,3 \mathrm{~m}$ & $103(53 \%)$ & $96(21 \%)$ \\
Número de plantas com altura $>1,3 \mathrm{~m}$ & $92(47 \%)$ & $371(79 \%)$ \\
Número plantas reprodutivas & \multicolumn{2}{c}{$35(18 \%)$} & \multicolumn{2}{c}{$226(48 \%)$} \\
Número de ramos médio & \multicolumn{2}{c}{1,1} & \multicolumn{2}{c}{1,6} \\
\hline & novas & mortas & novas & mortas \\
\hline 2005 & 13 & 9 & 1 & 6 \\
2006 & 1 & 7 & 1 & 5 \\
2007 & 9 & 16 & 0 & 5 \\
2008 & 1 & 14 & 3 & 12 \\
\hline Total & 24 & 46 & 5 & 28 \\
& $(12,3 \%)$ & $(23,6 \%)$ & $(1,1 \%)$ & $(6,0 \%)$ \\
\hline
\end{tabular}

$\mathrm{cm}$ considerando apenas as plantas reprodutivas (Tabela 2). A área basal (AB) média em 2004 foi de $0,495 \mathrm{~m}^{2}$ considerando todas as plantas com altura superior a $1,3 \mathrm{~m}$ e de $0,435 \mathrm{~m}^{2}$ considerando apenas as plantas reprodutivas (Tabela 2 ). A altura média considerando todas as plantas foi de $2,57 \mathrm{~m}, 4,19$ $\mathrm{m}$ considerando apenas as plantas com altura superior a 1,3 m, e 7,61 considerando apenas as plantas reprodutivas (Tabela 2 ).

Na FLONA Caçador, para o ano de 2004, o número total de plantas por hectare foi de 467 , sendo $371 \mathrm{com}$ altura $>1,3 \mathrm{~m}$, das quais 226 são reprodutivas, além de $96 \mathrm{com}$ altura $<1,3 \mathrm{~m}$. De 2004 a 2008 foram recrutadas 5 plantas e morreram 28 , conforme dinâmica expressa na Tabela 1. Das 28 plantas que morreram, sete tinham altura $>1,3$ metros (variando de 1,68 a 2,40 m), sendo as 21 restantes pertencentes a classe de regeneração natural ( $<1,3$ metros de altura).

O DAP médio foi de $4,77 \mathrm{~cm}$ considerando todas as plantas com altura superior a $1,3 \mathrm{~m}$, passíveis de serem avaliadas, e de 6,94 considerando apenas as plantas reprodutivas. A área basal ( $A B$ ) média em 2004 foi de 1,280 m² considerando todas as plantas com altura superior a $1,3 \mathrm{~m}$, e de $0,840 \mathrm{~m}^{2}$ considerando apenas as plantas reprodutivas (Tabela 2). A altura média considerando todas as plantas foi de $3,46 \mathrm{~m}, 4,07$ considerando apenas as plantas com altura superior a 1,3 m, e 4,96 considerando apenas as plantas reprodutivas. No Chile, esta espécie domina florestas secundárias, alcançando mais de $60 \%$ da área basal (Donoso et al., 2007) e valores de 5,2 $\mathrm{m}^{2}$ ha $^{-1}$ (Gutiérrez et al., 2004).

A dinâmica dos valores de DAP, altura e $A B$ ao longo dos anos estão expressas na Tabela 2 e na Figura 1, assim como a distribuição diamétrica e por classe de altura, além das taxas de incrementos para esses parâmetros. As equações de regressão para $D A P, A B$ e altura estão expressas na Tabela 3 . As distribuições diamétricas das duas áreas estudadas podem ser visualizadas nas Figuras 2 e 3, assim como a distribuição por classe de altura (Figuras 4 e 5).

Nas duas áreas as maiores freqüências de plantas estão na classe de DAP $0-5 \mathrm{~cm}$, equivalentes também em proporção (RGFC $=66 \%$ do total; FLONA $=72 \%$ do total). Porém, considerando apenas as plantas reprodutivas, na FLONA a proporção de plantas reprodutivas nesta classe em relação ao total é de $33 \%$, enquanto na RGFC esse valor é de $7 \%$, reflexo da entrada das plantas com menores dimensões em reprodução neste primeiro local.

$\mathrm{Na}$ distribuição por classe de altura, a RGFC apresenta $64 \%$ das plantas na classe $0-2$ $\mathrm{m}$, diminuindo a freqüência nas seguintes, apresentando plantas até a classe $18-20 \mathrm{~m}$, enquanto na FLONA a 0-2 m apresenta $28 \%$ das plantas, e a classe $2-4 \mathrm{~m}$ apresenta $38 \%$ das plantas, e a partir desta a freqüência diminui até a freqüência 8-10 m, limite superior da distribuição.

$\mathrm{Na}$ FLONA, sob o reflorestamento de Araucária, ocorreu um maior número de plantas, possivelmente devido a combinação de menor competição interespecífica e de maior luminosidade que chega ao sub-bosque da floresta, importante para o crescimento da espécie (Gottsberger et al., 1980). Armesto et al. (2001) e Guttiérrez et al. (2004) citam a espécie como intolerante a sombra. No Chile, esta espécie domina florestas secundárias, com alta incidência de luz, alcançando mais de $60 \%$ da área basal (Donoso et al., 2007) e valores de 5,2 $\mathrm{m}^{2} \mathrm{ha}^{-1}$ 
TABELA 2. Diâmetros à altura do peito (DAP), áreas basais (AB) e alturas médias de 2004 a 2008 das plantas de Drimys brasiliensis da Floresta Nacional de Caçador (FLONA) e da Reserva Genética Florestal de Caçador (RGFC).

\begin{tabular}{|c|c|c|c|c|c|}
\hline FLONA & 2004 & 2005 & 2006 & 2007 & 2008 \\
\hline \multicolumn{6}{|l|}{$\mathrm{DAP}(\mathrm{cm})$} \\
\hline$>1,3$ & $\begin{array}{c}4,77 \\
\left(2,91^{1}\right)\end{array}$ & $\begin{array}{c}4,99 \\
(2,99)\end{array}$ & $\begin{array}{c}5,12 \\
(3,06)\end{array}$ & $\begin{array}{c}5,35 \\
(3,14)\end{array}$ & $\begin{array}{c}5,45 \\
(3,17)\end{array}$ \\
\hline incremento & - & 0,22 & 0,13 & 0,23 & 0,10 \\
\hline Repr. & $\begin{array}{c}6,94 \\
(3,08)\end{array}$ & $\begin{array}{c}7,23 \\
(3,15)\end{array}$ & $\begin{array}{c}7,42 \\
(3,22)\end{array}$ & $\begin{array}{c}7,73 \\
(3,29)\end{array}$ & $\begin{array}{c}7,85 \\
(3,31)\end{array}$ \\
\hline incremento & - & 0,29 & 0,19 & 0,31 & 0,12 \\
\hline \multicolumn{6}{|l|}{$\mathrm{AB}\left(\mathrm{m}^{2}\right)$} \\
\hline$>1,3$ & 1,280 & 1,400 & 1,470 & 1,610 & 1,660 \\
\hline incremento & - & 0,120 & 0,070 & 0,140 & 0,050 \\
\hline reprod & 0,840 & 0,920 & 0,960 & 1,050 & 1,080 \\
\hline incremento & - & 0,080 & 0,040 & 0,090 & 0,030 \\
\hline \multicolumn{6}{|l|}{ Altura (m) } \\
\hline Total & $\begin{array}{c}3,46 \\
(2,04)\end{array}$ & $\begin{array}{c}3,61 \\
(2,06)\end{array}$ & $\begin{array}{c}3,80 \\
(2,16)\end{array}$ & $\begin{array}{c}3,98 \\
(2,26)\end{array}$ & $\begin{array}{c}4,14 \\
(2,31)\end{array}$ \\
\hline incremento & - & 0,15 & 0,19 & 0,18 & 0,16 \\
\hline$>1,3$ & $\begin{array}{c}4,07 \\
(1,82)\end{array}$ & $\begin{array}{c}4,23 \\
(1,82)\end{array}$ & $\begin{array}{c}4,44 \\
(1,92)\end{array}$ & $\begin{array}{c}4,62 \\
(2,03)\end{array}$ & $\begin{array}{c}4,77 \\
(2,09)\end{array}$ \\
\hline incremento & - & 0,16 & 0,21 & 0,18 & 0,15 \\
\hline Repr. & $\begin{array}{c}4,96 \\
(1,69)\end{array}$ & $\begin{array}{c}5,13 \\
(1,63)\end{array}$ & $\begin{array}{c}5,38 \\
(1,69)\end{array}$ & $\begin{array}{c}5,60 \\
(1,78)\end{array}$ & $\begin{array}{c}5,74 \\
(1,82)\end{array}$ \\
\hline incremento & - & 0,17 & 0,25 & 0,22 & 0,14 \\
\hline RGFC & 2004 & 2005 & 2006 & 2007 & 2008 \\
\hline \multicolumn{6}{|l|}{$\operatorname{DAP}(\mathrm{cm})$} \\
\hline$>1,3$ & $\begin{array}{c}7,94 \\
(6,12)\end{array}$ & $\begin{array}{c}8,06 \\
(6,20)\end{array}$ & $\begin{array}{c}8,13 \\
(6,23)\end{array}$ & $\begin{array}{c}8,18 \\
(6,22)\end{array}$ & $\begin{array}{c}8,14 \\
(6,21)\end{array}$ \\
\hline incremento & - & 0,12 & 0,07 & 0,05 & $-0,04$ \\
\hline Repr. & $\begin{array}{c}12,46 \\
(3,37)\end{array}$ & $\begin{array}{l}12,75 \\
(3,34)\end{array}$ & $\begin{array}{l}12,91 \\
(3,20)\end{array}$ & $\begin{array}{l}13,00 \\
(3,24)\end{array}$ & $\begin{array}{l}13,26 \\
(3,25)\end{array}$ \\
\hline incremento & - & 0,29 & 0,16 & 0,09 & 0,26 \\
\hline \multicolumn{6}{|l|}{$\mathrm{AB}\left(\mathrm{m}^{2}\right)$} \\
\hline$>1,3$ & 0,495 & 0,520 & 0,535 & 0,540 & 0,555 \\
\hline incremento & - & 0,025 & 0,015 & 0,005 & 0,015 \\
\hline Repr. & 0,435 & 0,455 & 0,465 & 0,465 & 0,475 \\
\hline $\begin{array}{l}1 \text { desvio padrão. } \\
\text { incremento. }\end{array}$ & - & 0,020 & 0,010 & 0,000 & 0,010 \\
\hline \multicolumn{6}{|l|}{ Altura (m) } \\
\hline Total & $\begin{array}{c}2,57 \\
(2,99)\end{array}$ & $\begin{array}{c}2,60 \\
(3,00)\end{array}$ & $\begin{array}{c}2,67 \\
(2,98)\end{array}$ & $\begin{array}{c}2,73 \\
(3,07)\end{array}$ & $\begin{array}{c}2,91 \\
(3,17)\end{array}$ \\
\hline incremento & - & 0,03 & 0,07 & 0,06 & 0,18 \\
\hline
\end{tabular}

(Gutiérrez et al., 2004). Devido a menor idade dessa população natural a mesma apresentou menores DAP médios e alturas médias, já que as plantas possuem no máximo 50 anos, pois no sub-bosque do reflorestamento de araucária na qual a população estudada se encontra foi implantado em 1958.

O valor de altura total na FLONA foi superior ao valor encontrado na RGFC considerando todas as plantas, pois na FLONA existe grande freqüência de plantas nas classes de altura acima de 2 metros, aumentando a média populacional, enquanto na RGFC a freqüência de plantas é maior na classe até 2 metros de altura (acima de $50 \%$ das plantas totais).

O comportamento descrito no parágrafo anterior possivelmente se deve a maior luminosidade que chega ao sub-bosque na FLONA, decorrente da inexistência da estratificação completa da floresta, onde as plantas de cataia existentes não precisam atravessar esses estratos para alcançar luminosidade, entrando inclusive em reprodução com menores dimensões. Também, possivelmente devido à menor idade da maioria das plantas adultas, pois no reflorestamento todas devem ter menos de 50 anos, enquanto na RGFC esta idade pode ser bastante superior. $\mathrm{Na}$ FLONA as plantas alcançaram no máximo 10,7 metros de altura e $19,3 \mathrm{~cm}$ de DAP, enquanto na RGFC as plantas alcançaram até 20 metros de altura e até $43 \mathrm{~cm}$ de DAP. Porém, devido ao maior número de plantas, a $A B$ na FLONA foi superior a da RGFC.

Essa hipótese de maior luminosidade e menor porte das plantas fica reforçada pela distribuição de plantas reprodutivas nas duas áreas. $\mathrm{Na}$ FLONA existem 226 plantas reproduzindo por hectare ( $48 \%$ das plantas totais), iniciando essa reprodução com $1,0 \mathrm{~cm}$ de DAP e $1,6 \mathrm{~m}$ de altura, enquanto na Reserva existem 35 plantas reproduzindo por hectare $(18 \%$ das plantas totais), iniciando essa reprodução com $1,6 \mathrm{~cm}$ de DAP e 2,7 metros de altura. Os tamanhos das plantas que iniciam a reprodução nas duas áreas estudadas não diferem muito numericamente, mas na FLONA na classe diamétrica até $5 \mathrm{~cm}$ de DAP existem 266 plantas, das quais 122 já estão reproduzindo (46\%), enquanto na RGFC na classe diamétrica até $5 \mathrm{~cm}$ de DAP existem 61 plantas, das quais apenas 6 já estão reproduzindo (10\%), diferindo estas freqüências estatisticamente $\left(X^{2}=\right.$ $4,615 ; g l=1 ; p<0,05)$. Possivelmente as plantas da FLONA têm um gasto energético menor com o crescimento para obter maior luminosidade, utilizando essa energia na reprodução, como verificado por Mariot et al. (2003) para Piper cernuum. 


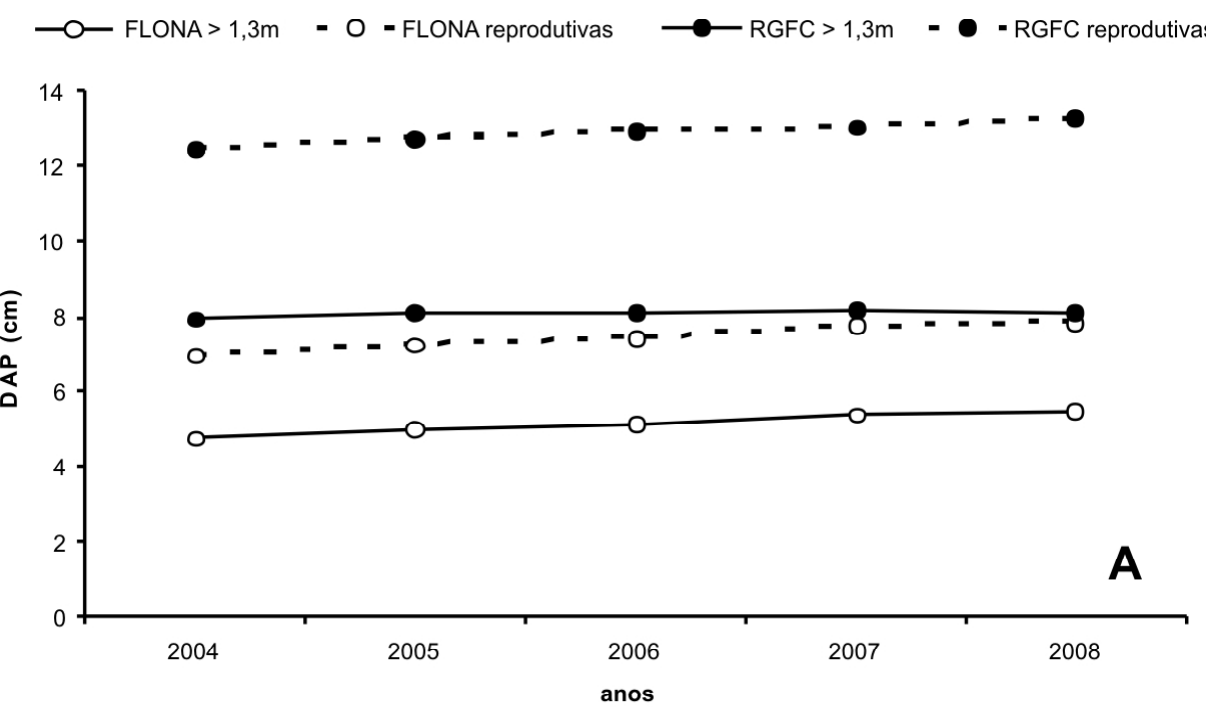

$\longrightarrow$-FLONA $>1,3 \mathrm{~m}-\mathrm{O}$-FLONA reprodutivas $\longrightarrow$ RGFC $>1,3 \mathrm{~m}-\bullet$ - RGFC reprodutivas

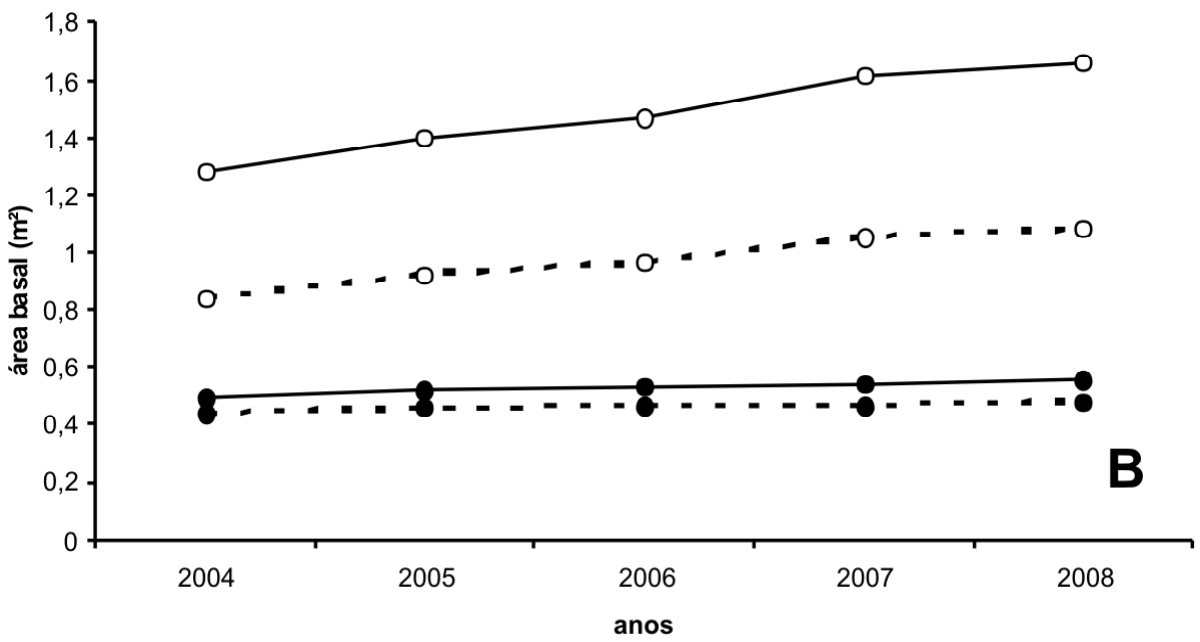

$\leadsto$ FLONA todas $\quad-0-$ FLONA $>1,3 \mathrm{~m} \quad \longrightarrow-$ FLONA reprodutivas

$\longrightarrow$ RGFC todas $\quad-\quad-$ RGFC $>1,3 m \quad \longrightarrow-$ RGFC reprodutivas

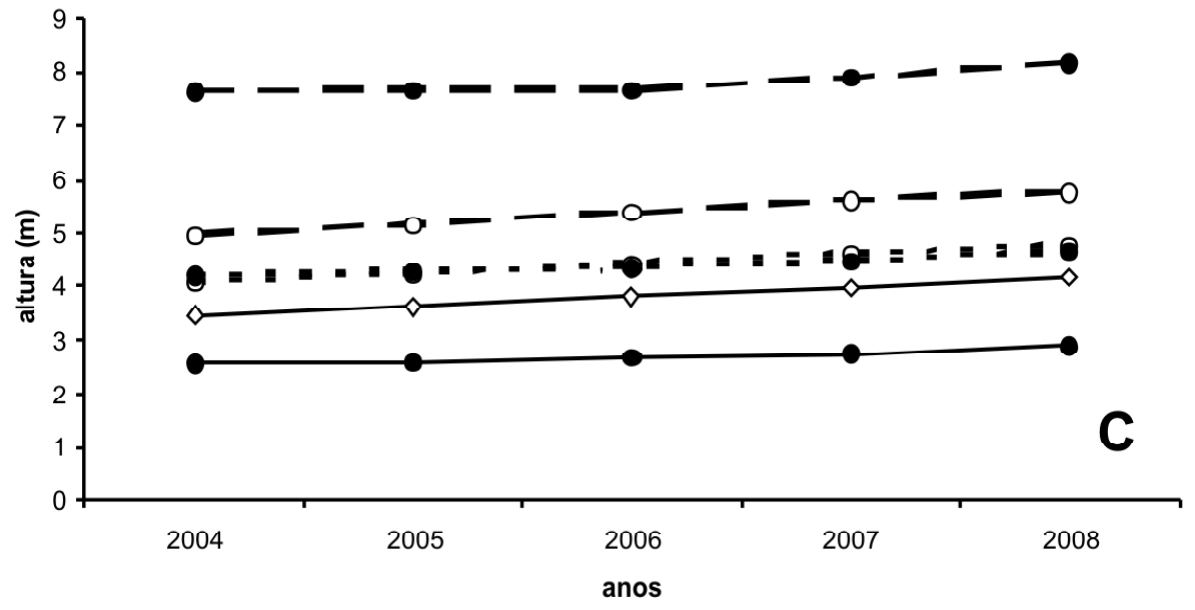

FIGURA 1. Dinâmica do Diâmetro à Altura do Peito (DAP-cm) $(\mathrm{A})$, Área Basal $\left(\mathrm{m}^{2}\right)(\mathrm{B})$ e Altura $(\mathrm{m})(\mathrm{C})$ de Drimys brasiliensis na Floresta Nacional de Caçador e na Reserva Genética Florestal de Caçador. 
TABELA3. Equações de regressão para os Incrementos Correntes Anuais (ICA) das variáveis (VAR) Diâmetro à Altura do Peito (DAP), Área Basal (AB) e Altura (ALT) de plantas de Drimys brasiliensis para os anos de 2005, 2006, 2007 e 2008 na Reserva Genética Florestal de Caçador (RGFC) e na Floresta Nacional de Caçador (FLONA).

\begin{tabular}{|c|c|c|c|c|c|}
\hline \multirow{2}{*}{$\frac{\text { VAR }}{\text { DAP }}$} & \multirow{2}{*}{$\frac{\text { ANO }}{2005}$} & \multicolumn{2}{|l|}{ RGFC } & \multicolumn{2}{|l|}{ FLONA Caçador } \\
\hline & & $0,0369^{* *} \mathrm{DAP}$ & $\mathrm{R}^{2}=0,82$ & $0,0923^{\star *} \mathrm{DAP}-0,0064^{\star *} \mathrm{DAP}^{2}$ & $\mathrm{R}^{2}=0,97$ \\
\hline & 2006 & $0,0228^{\star *} \mathrm{DAP}$ & $R^{2}=0,86$ & $0,0622^{* *}$ DAP $-0,0048^{* *} \mathrm{DAP}^{2}$ & $\mathrm{R}^{2}=0,96$ \\
\hline & 2007 & $0,0346^{* *} \mathrm{DAP}$ & $\mathrm{R}^{2}=0,82$ & $0,1356^{\star \star} \mathrm{DAP}-0,0115^{\star \star} \mathrm{DAP}^{2}$ & $\mathrm{R}^{2}=0,99$ \\
\hline & 2008 & $0,0305^{\star *} \mathrm{DAP}$ & $\mathrm{R}^{2}=0,81$ & $0,0662^{* *} \mathrm{DAP}-0,0058^{* *} \mathrm{DAP}^{2}$ & $\mathrm{R}^{2}=0,91$ \\
\hline \multirow[t]{4}{*}{$A B$} & 2005 & $0,000033^{* *}$ DAP & $\mathrm{R}^{2}=0,86$ & $0,000046^{* *} \mathrm{DAP}$ & $\mathrm{R}^{2}=0,97$ \\
\hline & 2006 & $0,000020^{* *}$ DAP & $\mathrm{R}^{2}=0,87$ & $0,000028^{\star *}$ DAP & $\mathrm{R}^{2}=0,96$ \\
\hline & 2007 & $0,000029^{\star *}$ DAP & $\mathrm{R}^{2}=0,85$ & $0,000052^{\star \star}$ DAP & $\mathrm{R}^{2}=0,95$ \\
\hline & 2008 & $0,000027^{\star \star}$ DAP & $\mathrm{R}^{2}=0,88$ & $0,000025^{\star \star} \mathrm{DAP}$ & $\mathrm{R}^{2}=0,91$ \\
\hline \multirow[t]{4}{*}{ ALT } & 2005 & $0,0812^{\star *} \mathrm{DAP}-0,0083^{* \star} \mathrm{DAP} 2$ & $R^{2}=0,82$ & $0,0938^{* *} \mathrm{DAP}-0,0106^{* \star} \mathrm{DAP}^{2}$ & $\mathrm{R}^{2}=0,92$ \\
\hline & 2006 & $0,0861^{* *} \mathrm{DAP}-0,0073^{\star} \mathrm{DAP}^{2}$ & $\mathrm{R}^{2}=0,77$ & $0,0725^{* *}$ DAP $-0,0047^{*}$ DAP $^{2}$ & $\mathrm{R}^{2}=0,95$ \\
\hline & 2007 & $0,0449^{* *} \mathrm{DAP}$ & $\mathrm{R}^{2}=0,88$ & $0,0415^{* *} \mathrm{DAP}$ & $\mathrm{R}^{2}=0,96$ \\
\hline & 2008 & $0,0339 *$ DAP & $R^{2}=0,84$ & $0,0335^{\star *} \mathrm{DAP}$ & $R^{2}=0,92$ \\
\hline
\end{tabular}

${ }^{*}=$ significativo ao nível de $5 \%$ de probabilidade; ${ }^{* *}=$ significativo ao nível de $1 \%$ de probabilidade

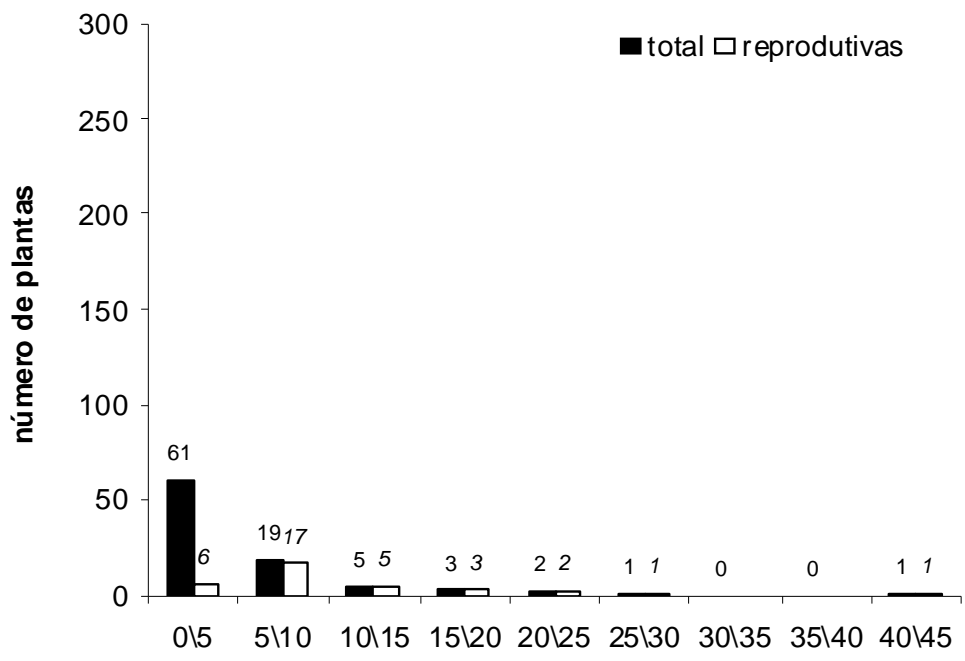

classes de DAP $(\mathrm{cm})$

FIGURA 2. Distribuiçāo diametrica de Drimys brasiliensis na Reserva Genética Florestal de Caçador, SC.

A menor quantidade de plantas na RGFC se deve a completa estratificação do ambiente florestal na área, resultando em maior diversidade de espécies (Negrelle \& Silva, 1992), ocasionando maior competição interespecífica, restando menos espaço para cada espécie. Na FLONA a diversidade é menor do que na RGFC, com dossel formado exclusivamente por Araucária, e um sub-bosque com pouca diversidade, com grande predominância de $D$. brasiliensis. Essa situação resultou em maiores incrementos em DAP, $A B$ e Altura na FLONA na maioria dos cortes nos diversos anos, e numa maior distribuição da espécie na área, como podem ser observados na Figura 1.

Para a RGFC o padrão espacial é agregado em todas as categorias analisadas e em todas as classes de distancias. Já para a FLONA verificase agregação com baixa intensidade apenas nas primeiras classes de distância (Figura 6). Em todas essas três categorias da FLONA a análise detectou 


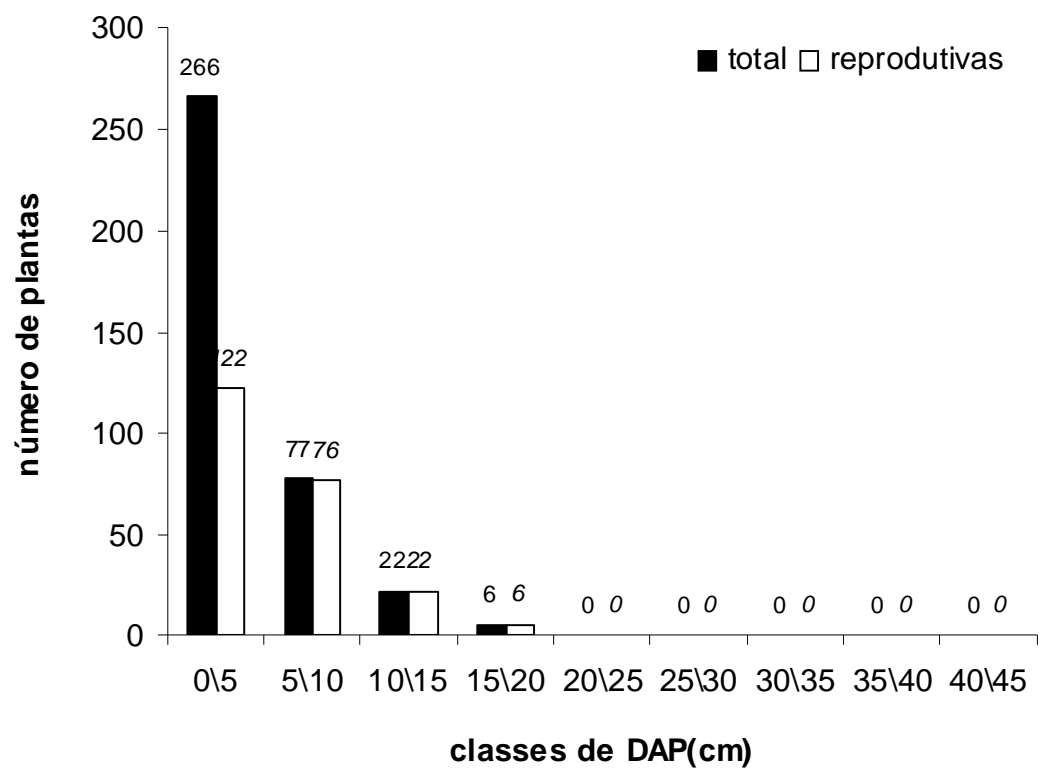

FIGURA 3. Distribuição diamétrica de Drimys brasiliensis na Floresta Nacional de Caçador, SC.

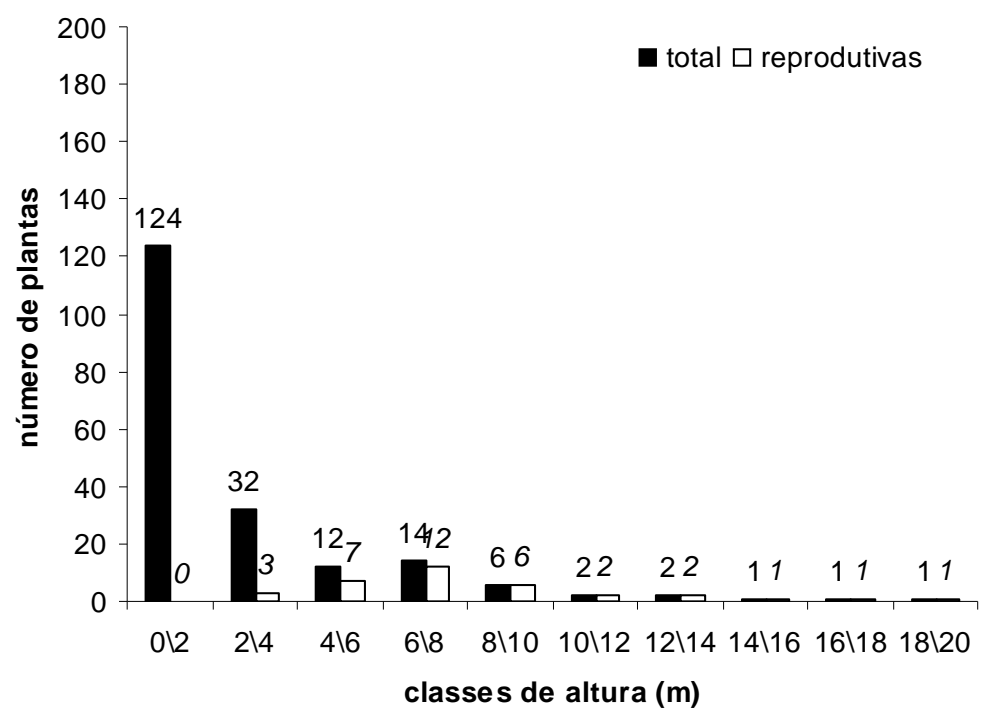

FIGURA4. Distribuição por classe de altura (metros) de Drimys brasiliensisna Reserva Genética Florestal de Caçador, SC.

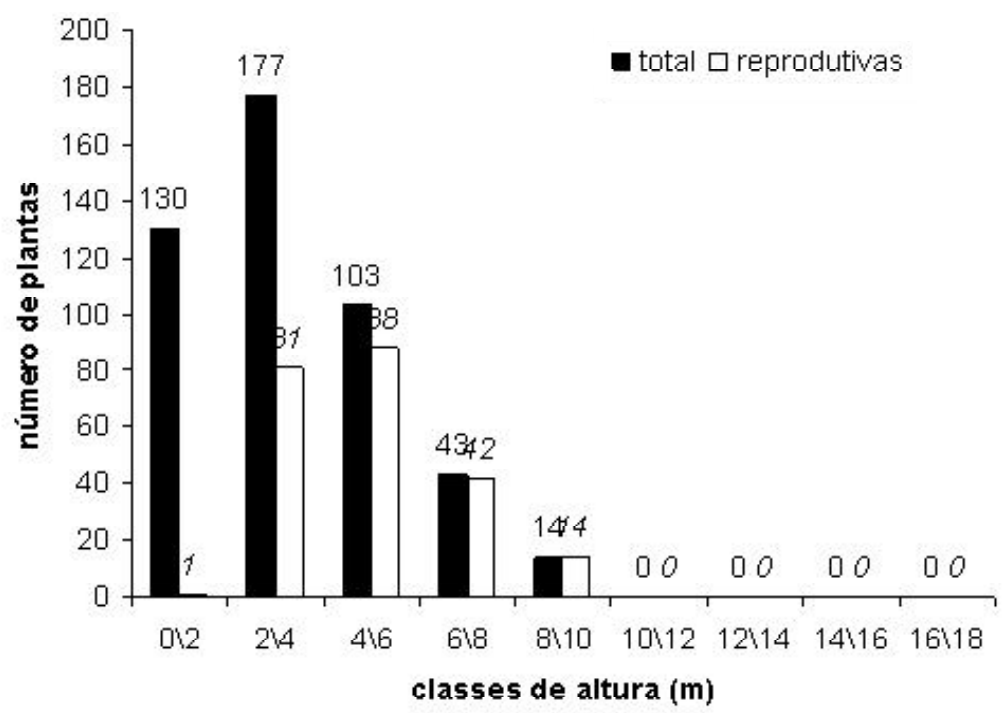

FIGURA 5. Distribuição por classe de altura (metros) de Drimys brasiliensis na Floresta Nacional de Caçador, SC. 

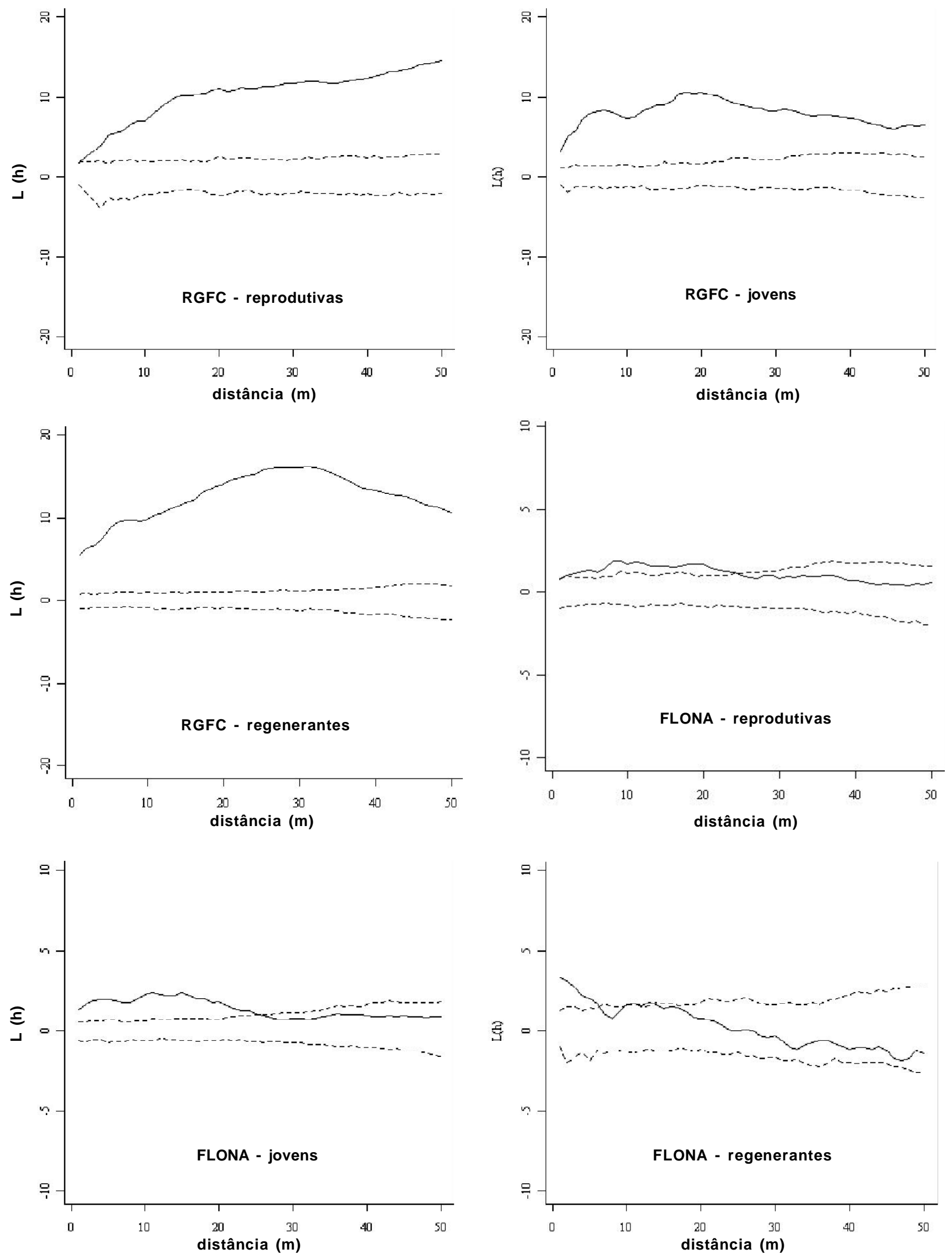

FIGURA 6. Padrão espacial de D. brasiliensis na Reserva Genética Florestal de Caçador (RGFC) e na Floresta Nacional de Caçador (FLONA). 
agregação com pouca intensidade. $D$. brasiliensis é dominante no sub-bosque em algumas florestas primárias, porém dependente de clareiras para a regeneração (Smith-Ramírez \& Amesto, 1994).

A FLONA, devido à maior luminosidade que chega ao solo, apresenta as condições necessárias para a regeneração de $D$. brasiliensis em toda a área, refletindo na aleatoriedade do padrão.o espacial. Na RGFC, possivelmente devido à floresta apresentar todos os estratos florestais, $D$. brasiliensis regenera em clareiras, refletindo no agrupamento das plantas em todas as coortes estudadas, reflexo das clareiras com as mais diversas idades de ocorrência.

Os estudos realizados possibilitaram o entendimento de diversos aspectos sobre a dinâmica demográfica de Drimys brasiliensis, que juntamente com outros estudos de autoecologia, possibilitarão a proposição de estratégia de manejo para a espécie em populações naturais, possibilitando renda a partir de recurso nãomadeireiro florestal.

Ambientes mais abertos ou secundários favoreceram a capacidade de incremento em diâmetro à altura do peito (DAP), altura $(\mathrm{H})$ e área basal $(A B)$, além de antecipar a reprodução em plantas de menor porte. Esse comportamento indica que a espécie possui potencial de manejo para obtenção de cascas em florestas secundárias, formação sucessional em que se encontra a maioria dos remanescentes de Mata Atlântica, além de indicar um potencial para cultivo em sistemas agroflorestais.

Drimys brasiliensis é apenas um produto que pode ser obtido da floresta como fonte de renda, incentivando a conservação dos fragmentos florestais da Mata Atlântica que restam. Existe diversidade muito grande de produtos florestais não-madeireiros que podem ser obtidos dessas áreas, como outras plantas medicinais (espinheira-santa e erva-mate), produtos comestíveis (pinhão, palmito e mel), plantas ornamentais (bromélias), entre outros, além dos produtos madeireiros, como fonte energética (lenha e carvão), além de matériaprima para construções e outras utilidades.

\section{REFERÊNCIA}

ARMESTO, J.J. et al. Seed rain of fleshy and dry propagules in different habitats in the temperate rainforests of Chiloé Island, Chile. Austral Ecology, v.26, p.311-20, 2001.

BACKES, P.; IRGANG, B. Árvores do Sul: guia de identificação \& interesse ecológico. Rio de Janeiro: Instituto Souza Cruz, 2002. 326p.
BALDAUF, C.; HANAZAKI, N.; REIS, M.S. Caracterização etnobotância dos sistemas de manejo de samambaiapreta (Rumohra adiantiformis (G. Forst) Ching Dryopteridaceae) utilizados no sul do Brasil. Acta Botanica Brasilica, v.21, n.4, p.823-34, 2007.

CECHINEL-FILHO, V. et al. Isolation and identification of active compounds from Drimys winteri barks. Journal of Ethnopharmacology, v.62, p.223-7, 1998.

CONTE, R. et al. Genetic structure and mating system of Euterpe edulis Mart. Populations: a comparative analysis using microsatellite and allozyme markers. Journal of Heredity, v.99, n.5, p.476-82, 2008.

DEGEN, B. et al. Impact of selective logging on genetic composition and demographic structure of four tropical tree species. Biological Conservation, v.131, p.386-401, 2006.

DONOSO, P.J.; SOTO, D.P.; BERTÍN, R.A. Size-density relationships in Drimys winteri secondary forests of the Chiloe Island, Chile: effects of physiography and species composition. Forest Ecology and Management, v.239, p.120-7, 2007.

DUARTE, A.S. et al. Estrutura demográfica e produção de frutos de Bromelia antiacantha Bertol. Revista Brasileira de Plantas Medicinais, v.9, n.3, p.106-12, 2007.

FANTINI, A.C. et al. Sustained yield management in tropical forest: a proposal based on the autoecology of species. Sellowia, v.42/44, p.25-33, 1992.

GOTTSBERGER, G.; SILBERBAER-GOTTSBERGER, I.; EHRENDORFER, F. Reproductive biology in the primitive relic Angiosperm Drimys brasiliensis (Winteraceae). Plant Systematic and Evolution, v.135, p.11-39, 1980.

GUTIÉRREZ, A.G.; ARMEStO, J.J.; ARAVENA, J.C. Disturbance and regeneration dynamics of an oldgrowth North Paragonian rain forest in Chiloé Island, Chile. Journal of Ecology, v.92, p.598-608, 2004.

HALL, P.; BAWA, K. Methods to asses the impact of extraction of non-timber tropical forest products on plant populations. Economic Botany, v.47, p.234-47, 1993. HERNÁNDEZ-APOLINAR, M.; VALVERDE, T.; PURATA, $S$. Demography of Bursera glabrifolia, a tropical tree used for folk woodcrafting in southern Mexico: An evaluation of its management plan. Forest Ecology and Management, v.223, p.139-51, 2006.

MALHEIROS, A. et al. Antifungal activity of drimane sesquiterpenes from Drimys brasiliensis using bioassay-guided fractionation. Journal of Pharmacy \&. Pharmaceutical Science, v.8, n.2, p.335-9, 2005. MARIOT, A.; DISTASI, L.C.; REIS, M.S. Genetic diversity in natural populations of Piper cernuum. Journal of Heredity, v.93, n.5, p.61-5, 2002.

MARIOT, A.; MANTOVANI, A.; REIS, M.S. Uso e conservação de Piper cernuum Vell. (Piperaceae) na Mata Atlântica: I. Fenologia reprodutiva e dispersão de sementes. Revista Brasileira de Plantas Medicinais, v.5, n.2, p.1-10, 2003.

MARIOT, A. et al. Uso e conservação de Piper cernuum Vell. (Piperaceae) na Mata Atlântica: II. Estrutura demográfica e potencial de manejo em floresta primária e secundária. Revista Brasileira de Plantas Medicinais, v.9, n.1, p.13-20, 2007.

NEGRELLE, R.A.B.; SILVA, F.C. Fitossociologia de um trecho de floresta com Araucaria angustifolia (Bert.)

Rev. Bras. PI. Med., Botucatu, v.12, n.2, p.168-178, 2010. 
O. Kuntze no município de Caçador-SC. Boletim de Pesquisa Florestal-EMBRAPA, v.24/25, p.37-54, 1992. PIO CORRÊA, M. Dicionário das plantas úteis do Brasil e das exóticas cultivadas. Rio de Janeiro: Imprensa Nacional, 1931, 6v, 707p.

PUCHALSKI, A.; MANTOVANI, M.; REIS, M.S. Variação em populações naturais de Araucaria angustifolia (Bert.) O. Kuntze associada a condições edafoclimáticas. Scientia Forestalis, v.70, p.137-48, 2006. REIS, M.S. Manejo sustentado de plantas medicinais em ecossistemas tropicais. In: DI STASI, L.C. (Ed.). Plantas medicinais: arte e ciência. Um guia de estudo interdisciplinar. São Paulo: UNESP, 1996. p.199-215.

REIS, M.S. et al. Management and conservation of natural populations in Atlantic rain Forest: the case study of plam heart (Euterpe edulis Martius). Biotropica, v.32, n.4B, p.894-902, 2000.

RIBEIRO, V.L.S. et al. Chemical composition and larvicidal properties of the essential oils from Drimys brasiliensis Miers (Winteraceae) on the cattle tick Rhipicephalus (Boophilus) microplus and the brown dog tick Rhipicephalus sanguineus. Parasitology Research, v.102, p.531-5, 2008.

RIPLEY, B.D. Modelling spatial patterns. Journal of the Royal Statistical Society, v.39, n.2, p.172-212, 1977. ROWLINGSON, B.; DIGGLE, P. Splancs: spatial point pattern analysis code in S-Plus. Computers and Geosciences, v.19, p.627-55, 1993.

RUSCHEL, A.R.; MOERSCHBACHER, B.M.; NODARI, R.O. Demography of Sorocea bonplandii in Seasonal
Deciduous Forest, Southern Brazil. Scientia Forestalis, v.70, p.149-59, 2006.

SEBBEN, A.M. et al. Modeling the long-term impacts of selective logging on genetic diversity and demographic structure of four tropical tree species in the Amazon forest. Forest Ecology and Management, v.254, p.335-49, 2008.

SIMÕES, C.M.O. et al. Plantas da medicina popular no Rio Grande do Sul. Porto Alegre: UFRGS, 1986. $147 p$.

SMITH-RAMÍREZ, C.; ARMESTO, J.J. Flowering and fruiting patterns in the temperate rainforest of Chiloé, Chile - ecologies and climatic constraints. Journal of Ecology, v.82, p.353-65, 1994.

STEENBOCK, W. et al. Avaliação de características fenotípicas para a determinação do rendimento foliar em espinheira-santa (Maytenus ilicifolia Martius). Revista Brasileira de Plantas Medicinais, v.6, n.1, p.71-6, 2003.

STEENBOCK, W.; REIS, M.S. Manejo de populações naturais de espinheira-santa. In: REIS, M.S.; SILVA, S.R. (Eds.). Conservação e uso sustentável de plantas medicinais e aromáticas: espinheira-santa. Brasília: Ibama, 2004. p.145-61.

TRINTA, E.F.; SANTOS, E. Flora llustrada Catarinense: Winteráceas. Itajaí: Herbário Barbosa Rodrigues, 1997. 19p.

WADT, L.H.O. et al. Sustainable forest use in Brazilian extractive reserves: Natural regeneration of Brazil nut in exploited populations. Biological Conservation, v.141, p.332-46, 2008. 